\title{
Incidencia de las características personales e inherentes al empleo sobre la satisfacción de los trabajadores en España*
}

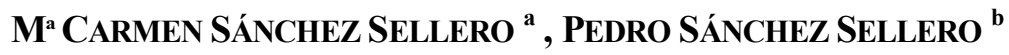 \\ a Universidad de La Coruña, Facultad de Economía y Empresa, Campus de Elviña, 15071 A Coruña, \\ España.E-mail: c.sanchez@udc.es \\ ${ }^{b}$ Universidad de Zaragoza, Escuela de Ingeniería y Arquitectura, Campus Río Ebro, 50018 \\ Zaragoza,España.E-mail: pedros@unizar.es
}

\begin{abstract}
RESUMEN
Con este trabajo se pretende determinar qué características afectan en mayor medida a la satisfacción de los trabajadores. El ámbito de aplicación es el mercado de trabajo español actual, aquejado de una fuerte recesión e inestabilidad. Se trata de averiguar si son las características personales de los trabajadores o si, por el contrario, son las características referidas a la organización las que influyen en mayor grado en la satisfacción laboral. Los datos provienen de la Encuesta de Calidad de Vida en el Trabajo, del Ministerio de Trabajo e Inmigración del año 2010. Mediante modelos lineales (ANOVA) se concluye que las variables inherentes al trabajo arrojan mejores resultados para explicar la satisfacción del trabajador, además, todos los coeficientes son positivos. Por último, se propone un modelo de regresión múltiple stepwise que permita jerarquizar la aportación de las distintas variables referidas al empleo sobre la satisfacción de los trabajadores; de todas ellas, la que tiene mayor incidencia es la motivación.
\end{abstract}

Palabras clave: Satisfacción laboral, remuneración, motivación, edad y género.

\section{Effect of the Personal and Job-Related Features on the Labour Satisfaction in Spain}

\begin{abstract}
This paper tries to determine the main features of the labour satisfaction. We applied the mentioned analysis to the current Spanish labour market, which is affected by a deep recession and instability. We want to get differences between personal and job-related features in order to find which better explain the labour satisfaction. Data come from the Quality of Labour Life Survey corresponding to the year 2010 by Ministry of Labour and Immigration of Spain. By means of linear models (ANOVA) we find that job-related variables explain better labour satisfaction, also, all the coefficients are positive. Finally, we propose a stepwise multiple regression which let us to put in order the effect of job-related variables on labour satisfaction. The variable with higher influence on labour satisfaction is motivation.
\end{abstract}

Keywords: Labour Satisfaction, Remuneration, Motivation, Age and Gender.

Clasificación JEL: J01, J21, J22, J28 y J30

\footnotetext{
* Este trabajo ha obtenido el Premio Asepelt Jóvenes Investigadores: Premio Bernardo Pena 2013 en el XXVII Congreso Internacional de Economía Aplicada Asepelt.
}

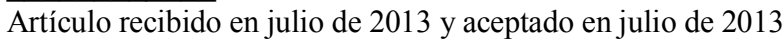

Artículo disponible en versión electrónica en la página www.revista-eea.net, ref. ə-32109 


\section{INTRODUCCIÓN}

La satisfacción de los trabajadores es un tema extensamente debatido tanto por economistas, como por sociólogos, psicólogos, y en cualquier área de conocimiento que estudie el ámbito laboral. Los factores que pueden influir en la satisfacción laboral son muchos, y muchos son también los autores que desde distintas vertientes analizan el grado de afectación de dichos factores sobre la satisfacción en el trabajo. La importancia que cada persona concede al trabajo en una escala con diferentes atributos es lo que se conoce como centralidad en el trabajo, concepto que guarda destacada relación con la satisfacción laboral.

Aunque el concepto de satisfacción en el trabajo se ha estudiado desde hace décadas, este artículo tiene la particularidad de ser analizado en el contexto del mercado laboral español actual, afectado por una muy larga fase de recesión económica.

Una primera versión de este trabajo se ha publicado en Sánchez Sellero y Sánchez Sellero (2013). Para el desarrollo de este trabajo se van a utilizar los microdatos de la Encuesta de calidad de vida en el trabajo (ECVT) del año 2010, elaborados por el Ministerio de Trabajo e Inmigración (últimos disponibles en julio de 2013). Para explicar el grado de satisfacción con el empleo actual se dispone de varias variables independientes (personales e inherentes al trabajo). Se van probando distintos modelos que se pueden clasificar en tres grupos: en un primer grupo se incluyen sólo las características personales, en un segundo grupo características propias de la organización, y en un tercer grupo, ambas características.

El objetivo inicial consiste en determinar entre los dos primeros grupos de variables señalados, cuál de ellos proporciona mejores resultados para explicar la satisfacción laboral. Para este fin, se emplea la modelización lineal general (ANOVA). Tras comprobar que las variables organizativas son las que explican mejor la satisfacción de los trabajadores, se plantea un segundo objetivo: mediante una regresión lineal múltiple stepwise y con 14 variables referidas al trabajo, se pretende averiguar el grado de influencia de cada una de ellas en la variable dependiente, estableciendo para ello una jerarquía entre esas variables, logrando un porcentaje de explicación muy bueno, del 63,4\%.

La estructura del artículo es la siguiente. En primer lugar, se revisan desde un punto de vista teórico los distintos factores que inciden en la satisfacción laboral. En segundo lugar, se describe la fuente de datos que proviene de la ECVT del año 2010. En tercer lugar, se desarrollan las diversas metodologías aplicadas: modelo lineal general (ANOVA) y regresión lineal múltiple stepwise. En base a estas herramientas estadísticas se van justificando distintos modelos que difieren básicamente en el tipo de variables incluidas, acompañándolos de comentarios a la vista de los resultados y de la idoneidad del modelo. En último término, se establecen las conclusiones del trabajo. 


\section{APROXIMACIONES TEÓRICAS}

La satisfacción laboral incide en la actitud del trabajador en el desarrollo de sus obligaciones laborales. La idea de satisfacción surge de la comparación entre el trabajo real y las expectativas que el trabajador se había generado. El trabajador estará insatisfecho si cree que está en desventaja respecto a sus compañeros, del mismo modo que si considera que el trabajo anterior le ofrecía mejores condiciones. A mayor satisfacción laboral, mayor compromiso del trabajador en el desempeño de sus tareas, y por tanto, mayor motivación; por el contrario, si la satisfacción en el trabajo es escasa o nula, el trabajador no tendrá motivaciones y no pondrá demasiado empeño en su actividad diaria.

La motivación laboral son los estímulos que posee una persona que lo conducen a actuar de peor o mejor manera en el ámbito laboral. Pueden venir del trabajo o de su círculo personal (familia, amigos). Pérez Rubio (1997) desde una perspectiva histórico-sociológica, estudia diferentes planteamientos en torno a la motivación y satisfacción laboral partiendo de las orientaciones, aspiraciones y expectativas del trabajador. Caballero (2002) expone que, en base a la conducta habida como consecuencia de la satisfacción en el trabajo, las relaciones que en el ámbito laboral se llevan a cabo y el nivel motivacional hacia el mismo, se generan distintas teorías de la motivación. Describe dos teorías que han contribuido al desarrollo de los modelos de la satisfacción en el trabajo:

1) "La teoría de los dos factores" de Herzberg et al. (1959). Establece que la satisfacción e insatisfacción laboral son dos fenómenos distintos y separados entre sí. Este modelo señala que el trabajador presenta dos grupos de necesidades: unas referidas al medio ambiente físico y psicológico del trabajo (necesidades higiénicas) y otras referidas al contenido mismo del trabajo (necesidades de motivación).

2) "El modelo de los determinantes de la satisfacción en el trabajo", propuesto por Lawler (1973) remarca la relación "expectativas-recompensas", desde distintas facetas y aspectos del trabajo. Se basa en la teoría de la motivación de Lawler y Porter (1967). La relación entre la expectativa y la recompensa obtenida produce la satisfacción o la insatisfacción laboral. Por ello, si la recompensa obtenida por el rendimiento en el trabajo excede de la que considera adecuada o si es equiparable, el trabajador alcanza el estado de satisfacción. Si esta relación se produce en sentido inverso, se produce la insatisfacción. En esta teoría, el término recompensa no solo se refiere a remuneración económica, sino que incluye un amplio abanico de resultados (sea reconocimientos, ascensos, valoración de superiores, etc.)

En el trabajo por cuenta ajena, a mayor confianza del trabajador con la empresa, mayor será la implicación de éste. Así lo reflejan distintas teorías de reciprocidad basadas en la percepción que tiene el trabajador del cumplimiento por 
parte de la empresa de las expectativas que éste tenía al principio. Los trabajadores que consideran que se han cumplido en mayor medida las promesas hechas por la empresa son: los que presentan una mayor centralidad del trabajo, los que han tenido un menor número de empleos, los que tienen un trabajo acorde con su cualificación, y quienes tienen además mayores niveles de implicación en el trabajo (Instituto Valenciano de Investigaciones Económicas, IVIE, 2007). En esta misma publicación se afirma que en cuanto a las características del empleo, los trabajadores con un contrato indefinido tienen una percepción más favorable de que las promesas realizadas fueron cumplidas. Por el contrario, quienes trabajan sin contrato, como en el empleo precario o en los trabajos familiares perciben un nivel de cumplimiento inferior. En base al tipo de empleador, quienes trabajan en la Administración o empresa pública perciben mayores niveles de cumplimiento que los que trabajan en la empresa privada.

En cuanto al concepto de centralidad del trabajo, y siguiendo a Alonso (2004), las áreas más importantes en la vida de las personas son: familia, pareja, amigos, trabajo, estudios, aficiones, voluntariado y religión. Para este autor, centralidad del trabajo es el orden otorgado por los individuos al trabajo. Se puede clasificar en tres niveles: centralidad alta (el trabajo es lo más importante, ocuparía el primero o segundo lugar), centralidad media (el trabajo ocupa el tercer o cuarto puesto en importancia), y centralidad baja (el trabajo ocupa el quinto o posterior lugar).

Bòria-Reverter et al. (2012) analizan los nexos de unión posibles entre salario, distintos activos intangibles y la satisfacción laboral en las organizaciones. Para estos autores, el concepto de clima organizacional se basa en atributos del entorno laboral. Si los empleados perciben un buen clima organizacional, sus comportamientos serán más adecuados para el logro del bienestar de la empresa. Para Cuadra-Peralta y Veloso-Besio (2010) los cimientos de un buen clima laboral se relacionan básicamente con el adecuado funcionamiento de la organización, y más específicamente con los siguientes indicadores: conciliación del trabajo con la vida familiar, prestaciones de tipo social, satisfacción en el puesto de trabajo y calidad directiva (liderazgo), entre otros. Robles-García et al. (2005) hablan de clima organizacional; para ellos, la satisfacción está fuertemente asociada a la valoración positiva de características propias de la organización.

Para Gamero (2005), la satisfacción en el trabajo es una variable susceptible de introducirse en los análisis económicos del mercado laboral. En la influencia de las características personales (edad, sexo, estudios) sobre la satisfacción laboral destaca el papel que juegan las expectativas laborales de las personas; cuanto menor sean las expectativas del trabajador, más probable será que se considere satisfecho. También afirma que la estabilidad laboral y las posibilidades de promoción tienen un fuerte impacto sobre la satisfacción laboral. Con 
respecto al estudio de los factores que explican la satisfacción laboral de los trabajadores asalariados en España, las características laborales con mayor efecto marginal sobre la probabilidad de estar muy satisfecho con el empleo actual son, por este orden, la tarea, la estabilidad del empleo, la dificultad para conciliar el ámbito laboral con el familiar, la percepción sobre la recompensa económica, y las relaciones con los jefes inmediatos. También repercute de manera considerable el entorno físico en el que se desarrolla la tarea y el desajuste con respecto a la carga de trabajo.

Gamero $(2007,2010)$ parte de dos hipótesis generales. Primero supone que un empleo no es sólo una combinación de salario y un número de horas de trabajo, hay otros factores relevantes. Como segunda hipótesis mantiene que los juicios de satisfacción laboral son una expresión que llama ex-post de la preferencia del trabajador por el empleo que ocupa actualmente respecto a otro que es "ideal". Este empleo de referencia reúne las características de lo que el individuo considera que es un "buen" empleo para sí mismo.

Bowling (2010) se refiere a algunos estudios de comportamiento que han examinado las interacciones entre la personalidad y las actitudes laborales.

En las investigaciones de Koustelios (2001) se examinó la relación entre las características personales y aspectos específicos de la satisfacción laboral. Okpara $(2004,2006)$ demuestra que las características personales como el sexo, edad, etc. son predictoras significativas de la satisfacción en el trabajo. En el trabajo de Clark et al. (2011) se expone que, en líneas generales, la satisfacción laboral aumenta linealmente con la edad; sin embargo, hay argumentos y evidencias empíricas que establecen que la relación tiene forma de $\mathrm{U}$, disminuyendo moderadamente en los primeros años de empleo y aumentando constantemente hasta su jubilación. Por tanto, evidencian una relación en forma de U entre la edad y la satisfacción laboral. Según Allen y Van der Velden (2001) la educación repercute sobre los salarios y otros resultados del mercado laboral; para estos autores las distintas habilidades son mucho mejores predictores de la satisfacción en el trabajo que el nivel educativo.

En Judge et al. (2001) se realiza una revisión cualitativa y cuantitativa de la relación entre la satisfacción laboral y el desempeño laboral. En la investigación empírica de Lund (2003) se analiza el impacto de los tipos de cultura organizacional en la satisfacción laboral.

En el trabajo de Gazioglu y Tansel (2006), cuatro medidas diferentes de satisfacción en el trabajo están relacionadas con una variedad de características personales y laborales. Analizan la satisfacción laboral con respecto a la composición industrial y ocupaciones. Concluyen que los sectores de educación y sanidad están menos satisfechos con sus salarios pero más satisfechos con su sentido de logro. Además, los empleados que recibieron capacitación para el trabajo estaban más satisfechos que los que no tuvieron oportunidades de for- 
mación. A diferencia de estudios anteriores, se encontró que las personas casadas tienen niveles más bajos de satisfacción laboral que las no casadas. Otros resultados confirman los de la literatura, como que las mujeres están más satisfechas que los hombres, y una relación en forma de $\mathrm{U}$ entre satisfacción y edad.

Donohue y Heywood (2004) analizan la satisfacción laboral en función del género. Westover (2012a) establece que del mismo modo que la satisfacción laboral afecta a una gran variedad de resultados sociales, las organizaciones deben reconocer las diferencias de género en este concepto. En muchas investigaciones se constata que las mujeres presentan un nivel superior de satisfacción laboral al de los varones. El trabajo de Sánchez et al. (2007) confirma este resultado. Se revisan las diferencias entre hombres y mujeres respecto a la influencia de otras variables sociodemográficas, del puesto y de la organización sobre la satisfacción con el trabajo.

Para Cohrs et al. (2006), la satisfacción en el trabajo puede ser conceptualizada como una función de las condiciones situacionales, las características personales y las interacciones entre ambos grupos de variables. Franek y Vecera (2008) examinan la relación entre la satisfacción laboral y una selección de variables personales en un grupo de personas pertenecientes a distintas áreas ocupacionales.

López-Araújo et al. (2007) concluyen que cuando los niveles de las dos dimensiones de la implicación en el trabajo - identificación psicológica y sentimientos de deber - obligación hacia el trabajo - son elevados, el estrés, por los logros conseguidos y el desarrollo de la carrera, no influye sobre la satisfacción laboral. Sin embargo, aunque el estrés sea alto, cuando la identificación psicológica es baja y los sentimientos de deber-obligación hacia el trabajo son elevados, se observa una mayor satisfacción con el trabajo. Para Zeytinoglu et al. (2007) la intensificación del trabajo contribuye a incrementar el estrés y disminuir la satisfacción laboral.

Petrescu y Simmons (2008) investigan la relación entre la gestión de recursos humanos y la satisfacción de los trabajadores con su remuneración. Centran su estudio en el impacto sobre la satisfacción laboral general y la satisfacción con la remuneración. Singh y Loncar (2010) examinan las relaciones entre satisfacción con el salario, satisfacción con el empleo y cambio de empleo. Los resultados del estudio de Tremblay et al. (2012) demostraron que las motivaciones familiares y aquellas vinculadas al desarrollo profesional tienen un efecto positivo sobre la satisfacción; únicamente las buenas condiciones económicas no resultan suficientes.

De los resultados de Gómez (2010) se desprende que los empleados temporales presentan una mediana satisfacción frente a necesidades de seguridad, fisiológicas, y afiliación; e insatisfacción en las necesidades de autorrealización, 
reconocimiento, creatividad y de descanso. Los resultados reflejan un panorama sombrío sobre la calidad de vida laboral que obtiene este tipo de empleados.

El propósito de Westover y Taylor (2010) es explorar las diferencias entre distintos países en las satisfacciones del trabajo y sus determinantes en el tiempo. En un mercado global cada vez más competitivo, las organizaciones se preguntan cómo sacar más provecho de sus empleados. Las distintas soluciones pasan por el fortalecimiento y la difusión de los valores fundamentales de la organización, y la creencia de que un aumento de la satisfacción laboral produce beneficios en la productividad del trabajador, y ayuda a crear un compromiso de alto rendimiento. Para Westover (2012b), la satisfacción laboral es un concepto dinámico que cambia en respuesta a las condiciones personales y ambientales. Es a través del tiempo y en diferentes contextos como se examinan y comprenden mejor los factores principales que afectan a la satisfacción laboral.

En un sentido más amplio, Rode (2004) afirma que la satisfacción laboral se relaciona significativamente con la satisfacción vital. Examina la relación entre trabajo y satisfacción con la vida, así como satisfacción no laboral y variables ambientales. Del Líbano et al. (2005) constatan una relación negativa entre la adicción al trabajo y la satisfacción extra-laboral, es decir, que los adictos al trabajo son los menos satisfechos fuera de su vida laboral.

Desde otras vertientes, Barmby et al. (2012) tratan el capital humano y la satisfacción laboral. Khanin et al. (2012) estudian cómo aumentar la satisfacción en el trabajo y reducir las intenciones de rotación en la empresa familiar.

\section{FUENTE DE DATOS}

Los análisis que se exponen en este estudio se basan en los microdatos de la Encuesta de calidad de vida en el trabajo (ECVT) facilitados por el Ministerio de Trabajo e Inmigración y correspondientes al año 2010. El ámbito geográfico de esta encuesta es todo el territorio español, exceptuando a Ceuta y Melilla. El ámbito poblacional está delimitado por la población ocupada de 16 y más años que reside en viviendas familiares.

\section{MÉTODOS Y VARIABLES}

Los resultados del estudio de Mpeka (2012) demostraron que los compañeros de trabajo, remuneración, promoción, supervisión y trabajo en sí, tienen una influencia significativa en la satisfacción en el trabajo, mientras que la edad y el género no. Por lo tanto, se deben promover activamente esos factores en el lugar de trabajo con el fin de promover un mejor desempeño en el trabajo. Se recomienda el uso de variables sociológicas más amplias para identificar las variables adicionales que son vitales en la creación de un lugar de trabajo atractivo. 
En el modelo de Ellickson y Logsdon (2001) se analiza la influencia de 11 variables ambientales y 3 factores demográficos sobre la satisfacción laboral en un grupo de 1200 trabajadores. Un análisis de regresión revela que los factores ambientales tales como oportunidades de promoción, satisfacción con la remuneración, etc. son significativos (y positivos) en relación con la satisfacción laboral global. Sin embargo, las variables demográficas son pobres predictoras de la satisfacción laboral. El modelo explica más del $50 \%$ de la variación en la satisfacción laboral de los trabajadores estudiados.

En parecidos términos se argumenta este trabajo, que se presenta seguidamente. Aunque la encuesta la forman 8061 personas, al eliminar la falta de respuesta en algunas preguntas, queda en 5939 (en los primeros modelos). Para extender los resultados a la población, se ha ponderado por los factores de elevación correspondientes. La metodología utilizada, tanto la relativa a los modelos lineales generales (ANOVA) como la regresión lineal múltiple stepwise, se justifica no solo por el tipo de variables implicadas sino también por la propia finalidad del estudio. A lo largo de la exposición de estos modelos también se irá comprobando algunos resultados que confirman la idoneidad de los métodos aplicados.

Tabla 1

Modelos y variables incluidas

\begin{tabular}{|c|c|c|}
\hline Modelos & Variables & $\mathbf{R}$ cuadrado \\
\hline Modelos grupo 1 & $\begin{array}{l}\text { Sexo } \\
\text { Edad (categórica) } \\
\text { Nivel de estudios }\end{array}$ & $\begin{array}{l}0,015^{a} \\
0,042^{b}\end{array}$ \\
\hline Modelo grupo 2 & $\begin{array}{l}\text { GSmotivación } \\
\text { GSactividad } \\
\text { GSorg.trabajo } \\
\text { GSsalario }\end{array}$ & 0,588 \\
\hline Modelos grupo 3 & $\begin{array}{l}\text { Sexo } \\
\text { Edad (categórica) } \\
\text { Nivel de estudios } \\
\text { GSmotivación } \\
\text { GSactividad } \\
\text { GSorg.trabajo } \\
\text { GSsalario }\end{array}$ & $\begin{array}{l}0,591^{\mathrm{c}} \\
0,593^{\mathrm{d}} \\
0,605^{\mathrm{e}}\end{array}$ \\
\hline
\end{tabular}

Nota: a. modelo personalizado (con efectos principales e interacciones del sexo con las demás variables cualitativas). b. modelo factorial (con todas las interacciones). c. modelo de efectos principales. d. modelo personalizado (con efectos principales e interacciones del sexo con las demás variables cualitativas). e. modelo factorial (con todas las interacciones).

Fuente: Elaboración propia a partir de ECVT (Ministerio de Trabajo e Inmigración, 2010).

La variable dependiente es el grado de satisfacción con el trabajo actual 
(GStrabajoactual) y las variables independientes son: sexo, edad (categórica), nivel de estudios (todas éstas, variables cualitativas) y grado satisfacción-motivación (GSmotivación), grado satisfacción actividad (GSactividad), grado satisfacción organización trabajo (GSorg.trabajo) y grado satisfacción salario (GSsalario) (todas ellas cuantitativas, toman valores de 0 a 10). En la Tabla 1 figuran los modelos que se van a desarrollar (modelos lineales generales, ANOVA), y en la Tabla 2 la descripción de las variables. En los modelos del grupo 1 las variables independientes son las características personales, en el modelo del grupo 2 figuran las características propias del trabajo, y en los modelos del grupo 3 se incluyen ambos tipos de variables.

\section{Tabla 2}

Variables utilizadas en los modelos y sus categorías o valores

\begin{tabular}{|c|c|}
\hline Variables & Categorías o valores \\
\hline Sexo & $\begin{array}{l}\text { 1. Varón } \\
\text { 2. Mujer }\end{array}$ \\
\hline Edad (categórica) & $\begin{array}{l}\text { 1. } 16-25 \text { años } \\
\text { 2. } 26-35 \text { años } \\
\text { 3. } 36-45 \text { años } \\
\text { 4. } 46-55 \text { años } \\
\text { 5. } 56-65 \text { años, } \\
\text { 6. más de } 65 \text { años }\end{array}$ \\
\hline Nivel de estudios & $\begin{array}{l}\text { 1. No sabe leer ni escribir } \\
\text { 2. Menos que estudios primarios } \\
\text { 3. Enseñanza primaria } \\
\text { 4. Enseñanza secundaria } \\
\text { 5. Ciclos formativos de grado medio (Formación profesional FPI) } \\
\text { 6. Ciclos formativos de grado superior (Formación profesional FPII) } \\
\text { 7. Bachiller superior, BUP, COU o equivalentes } \\
\text { 8. Estudios universitarios de grado medio } \\
\text { 9. Estudios universitarios de grado superior de segundo o tercer ciclo }\end{array}$ \\
\hline GSmotivación & De 0 a10 \\
\hline GSactividad & De 0 a10 \\
\hline GSorg.trabajo & De 0 a10 \\
\hline GSsalario & De 0 a10 \\
\hline
\end{tabular}

Fuente: Elaboración propia a partir de ECVT (Ministerio de Trabajo e Inmigración, 2010).

\section{DISCUSIÓN}

En la Tabla 1 se pone de manifiesto que los modelos del grupo 1 no sirven para explicar la variación en la satisfacción laboral de los trabajadores $(1,5 \%$ y $4,2 \%$ es el porcentaje explicado, dependiendo de si el modelo es de efectos principales con todas o algunas interacciones). En esta tabla se observa que mientras las características personales (modelos grupo 1) explican entre 1-4\% 
aproximadamente de la satisfacción laboral, las características propias del trabajo (modelo grupo 2) explican más de un $50 \%$.

Tabla 3

Resumen del modelo, estimaciones del tamaño de los efectos (modelo grupo $3^{d}$ )

\begin{tabular}{|l|r|r|r|r|r|r|}
\hline \multicolumn{1}{|c|}{ Fuente } & $\begin{array}{c}\text { Suma de } \\
\text { cuadrados } \\
\text { tipo III }\end{array}$ & gl & \multicolumn{1}{c|}{$\begin{array}{c}\text { Media } \\
\text { cuadrática }\end{array}$} & F & $\begin{array}{c}\text { Eta } \\
\text { cuadrado } \\
\text { parcial }\end{array}$ \\
\hline Modelo corregido & $2,561 \mathrm{E} 7$ & 31 & 826162,52 & 653457,416 & 0,00 & 0,593 \\
\hline Intersección & 501054,33 & 1 & 501054,33 & 396311,455 & 0,00 & 0,028 \\
\hline Sexo & 15,945 & 1 & 15,945 & 12,612 & 0,00 & 0,000 \\
\hline Edad categórica & 54387,553 & 5 & 10877,511 & 8603,622 & 0,00 & 0,003 \\
\hline Nivel de estudios & 30842,162 & 8 & 3855,270 & 3049,345 & 0,00 & 0,002 \\
\hline GSmotivación & 2090535 & 1 & 2090535 & 1653519,19 & 0,00 & 0,106 \\
\hline GSactividad & 1971505,7 & 1 & 1971505,7 & 1559372,4 & 0,00 & 0,101 \\
\hline GSorg.trabajo & 1236522,7 & 1 & 1236522,7 & 978033,876 & 0,00 & 0,066 \\
\hline GSsalario & 1329744,1 & 1 & 1329744,1 & 1051767,790 & 0,00 & 0,070 \\
\hline Sexo * Edad categórica & 18601,328 & 5 & 3720,266 & 2942,563 & 0,00 & 0,001 \\
\hline Sexo * Nivel de estudios & 91276,045 & 8 & 11409,506 & 9024,406 & 0,00 & 0,005 \\
\hline Error & $1,757 \mathrm{E} 7$ & 13893146 & & 1,264 & & \\
\hline Total & $7,951 \mathrm{E} 8$ & 13893178 & & & & \\
\hline Total corregida & $4,318 \mathrm{E} 7$ & 13893177 & & & \\
\hline
\end{tabular}

Nota: $d$. modelo personalizado (con efectos principales e interacciones del sexo con las demás variables cualitativas.

Variable dependiente: GStrabajoactual. $R$ cuadrado $=0,593$ ( $R$ cuadrado corregida $=0,593$ )

Fuente: Elaboración propia a partir de ECVT (Ministerio de Trabajo e Inmigración, 2010).

De los modelos del grupo 3, en el que intervienen características personales y propias del trabajo, desarrollamos en la Tabla 3 el modelo con efectos principales e interacciones del sexo con las otras dos variables cualitativas (el género influye en gran medida en distintos indicadores del mercado laboral ${ }^{1}$ ), $\mathrm{R}^{2}=0,593$. Elegimos este modelo, aunque poca diferencia hay en la bondad del modelo con los demás de ese grupo, porque en el modelo factorial las salidas informáticas de resultados son demasiado extensas. En la Tabla 3 también aparecen las estimaciones del grado en que cada factor o combinación de factores está afectando a la variable dependiente. El estadístico eta cuadrado parcial, que para un efecto A se obtiene de la siguiente manera: $\left(\mathrm{F}_{\mathrm{A}} * \mathrm{gl}_{\mathrm{A}}\right) /\left(\mathrm{F}_{\mathrm{A}} *\right.$ $\mathrm{gl}_{\mathrm{A}}+\mathrm{gl}_{\text {error }}$ ), se interpreta como la proporción de la varianza explicada, es decir, es una estimación de la proporción de la variación de la variable dependiente

\footnotetext{
${ }^{1}$ A modo de ejemplo, en el trabajo de Sánchez Sellero (2012) se plantean tres modelos en el mercado laboral, en los que todas las variables se crean en función del género de la persona analizada.
} 
que está explicada por cada efecto. En este caso, se observa que los valores más altos de este estadístico corresponden a las variables GSmotivación, GSactividad, GSorg.trabajo y GSsalario, con lo cual, son los factores que afectan en mayor grado a la explicación de la satisfacción laboral (GStrabajoactual).

En la Tabla 4 aparecen las estimaciones de los parámetros del modelo anterior. Puesto que las estimaciones de los parámetros suman cero para cada efecto, la tabla no recoge las estimaciones que son redundantes. Todos los coeficientes son significativos. Por el signo de los parámetros se concluye que en cuanto al sexo, las mujeres tienen signo positivo en satisfacción laboral (mayor que los hombres, que aparecen con signo negativo). A la vista de los parámetros de la variable edad asemejan forma de U (como señala la literatura), y en cuanto al nivel de estudios no hay un patrón claro. Los coeficientes de las variables cuantitativas, que son las características de la organización (GSmotivación, GSactividad, GSorg.trabajo y GSsalario) son todos positivos, por lo que al aumentar por ejemplo, en un punto el grado de satisfacción con la actividad el grado de satisfacción con el trabajo actual aumenta 0,281 puntos, manteniendo constantes las demás variables.

\section{Tabla 4}

Estimaciones de los parámetros (modelo grupo $3^{\mathrm{d}}$ )

\begin{tabular}{|c|c|c|c|c|c|c|}
\hline \multirow[b]{2}{*}{ Parámetro } & \multirow[b]{2}{*}{ B } & \multirow{2}{*}{$\begin{array}{l}\text { Error } \\
\text { típ. }\end{array}$} & \multirow[b]{2}{*}{$\mathbf{t}$} & \multirow[b]{2}{*}{ Sig. } & \multicolumn{2}{|c|}{ Intervalo de confianza al $95 \%$} \\
\hline & & & & & $\begin{array}{l}\text { Límite } \\
\text { inferior }\end{array}$ & $\begin{array}{l}\text { Límite } \\
\text { superior }\end{array}$ \\
\hline Intersección & 1,852 & 0,009 & 199,815 & 0,000 & 1,834 & 1,871 \\
\hline$[\operatorname{Sexo}=1,00]$ & $-0,194$ & 0,018 & $-10,534$ & 0,000 & $-0,230$ & $-0,158$ \\
\hline$[\operatorname{Sexo}=2,00]$ & $0^{\mathrm{a}}$ & - & - & - & - & - \\
\hline [Edadcategorica=1,00] & $-0,547$ & 0,009 & $-59,510$ & 0,000 & $-0,565$ & $-0,529$ \\
\hline [Edadcategorica=2,00] & $-0,290$ & 0,009 & $-31,794$ & 0,000 & $-0,307$ & $-0,272$ \\
\hline [Edadcategorica $=3,00]$ & $-0,263$ & 0,009 & $-28,875$ & 0,000 & $-0,281$ & $-0,245$ \\
\hline [Edadcategorica $=4,00]$ & $-0,329$ & 0,009 & $-36,054$ & 0,000 & $-0,347$ & $-0,311$ \\
\hline [Edadcategorica $=5,00]$ & $-0,175$ & 0,009 & $-18,931$ & 0,000 & $-0,193$ & $-0,156$ \\
\hline [Edadcategorica $=6,00]$ & $0^{\mathrm{a}}$ & - & - & - & - & - \\
\hline [Niveldeestudios $=1,00$ ] & 0,518 & 0,015 & 34,557 & 0,000 & 0,489 & 0,547 \\
\hline [Niveldeestudios $=2,00$ ] & 0,334 & 0,005 & 67,548 & 0,000 & 0,324 & 0,343 \\
\hline [Niveldeestudios $=3,00$ ] & $-0,043$ & 0,002 & $-23,111$ & 0,000 & $-0,047$ & $-0,040$ \\
\hline [Niveldeestudios $=4,00]$ & 0,075 & 0,002 & 48,646 & 0,000 & 0,072 & 0,078 \\
\hline [Niveldeestudios $=5,00]$ & $-0,054$ & 0,002 & $-30,253$ & 0,000 & $-0,057$ & $-0,050$ \\
\hline [Niveldeestudios $=6,00]$ & $-0,075$ & 0,002 & $-43,699$ & 0,000 & $-0,079$ & $-0,072$ \\
\hline [Niveldeestudios $=7,00]$ & 0,004 & 0,002 & 2,546 & 0,011 & 0,001 & 0,007 \\
\hline [Niveldeestudios $=8,00]$ & $-0,050$ & 0,002 & $-32,772$ & 0,000 & $-0,053$ & $-0,047$ \\
\hline
\end{tabular}


Tabla 4 (continuación)

Estimaciones de los parámetros (modelo grupo $3^{\mathrm{d}}$ )

\begin{tabular}{|c|c|c|c|c|c|c|}
\hline \multirow{2}{*}{ Parámetro } & \multirow{2}{*}{ B } & \multirow{2}{*}{$\begin{array}{l}\text { Error } \\
\text { típ. }\end{array}$} & \multirow{2}{*}{$\mathbf{t}$} & \multirow{2}{*}{ Sig. } & \multicolumn{2}{|c|}{$\begin{array}{c}\text { Intervalo de confianza al } \\
95 \%\end{array}$} \\
\hline & & & & & $\begin{array}{l}\text { Límite } \\
\text { inferior }\end{array}$ & $\begin{array}{l}\text { Límite } \\
\text { superior }\end{array}$ \\
\hline [Niveldeestudios $=9,00]$ & $0^{\mathrm{a}}$ & - & - & - & - & - \\
\hline $\begin{array}{l}{[\text { Sexo }=1,00]^{*}} \\
{[\text { Edadcategorica }=1,00]}\end{array}$ & 0,323 & 0,018 & 17,470 & 0,000 & 0,287 & 0,359 \\
\hline $\begin{array}{l}{[\text { Sexo }=1,00]^{*}} \\
{[\text { Edadcategorica }=2,00]}\end{array}$ & 0,086 & 0,018 & 4,644 & 0,000 & 0,049 & 0,122 \\
\hline $\begin{array}{l}{[\text { Sexo }=1,00]^{*}} \\
{[\text { Edadcategorica }=3,00]}\end{array}$ & 0,082 & 0,018 & 4,436 & 0,000 & 0,046 & 0,118 \\
\hline $\begin{array}{l}{[\text { Sexo }=1,00]^{*}} \\
{[\text { Edadcategorica }=4,00]}\end{array}$ & 0,175 & 0,018 & 9,482 & 0,000 & 0,139 & 0,211 \\
\hline $\begin{array}{l}{[\text { Sexo }=1,00]{ }^{*}} \\
{[\text { Edadcategorica }=5,00]}\end{array}$ & 0,178 & 0,018 & 9,602 & 0,000 & 0,141 & 0,214 \\
\hline $\begin{array}{l}{[\text { Sexo }=1,00]^{*}} \\
{[\text { Edadcategorica }=6,00]}\end{array}$ & $0^{a}$ & - & - & - & - & - \\
\hline $\begin{array}{l}{[\text { Sexo }=2,00]^{*}} \\
{[\text { Edadcategorica }=1,00]}\end{array}$ & $0^{a}$ & - & - & - & - & - \\
\hline $\begin{array}{l}{[\text { Sexo }=2,00]{ }^{*}} \\
{[\text { Edadcategorica }=2,00]}\end{array}$ & $0^{a}$ & - & - & - & - & - \\
\hline $\begin{array}{l}{[\text { Sexo }=2,00]^{*}} \\
{[\text { Edadcategorica }=3,00]}\end{array}$ & $0^{a}$ & - & - & - & - & - \\
\hline $\begin{array}{l}{[\text { Sexo }=2,00]{ }^{*}} \\
{[\text { Edadcategorica }=4,00]}\end{array}$ & $0^{a}$ & - & - & - & - & - \\
\hline $\begin{array}{l}{[\text { Sexo }=2,00]{ }^{*}} \\
{[\text { Edadcategorica }=5,00]}\end{array}$ & $0^{a}$ & - & - & - & - & - \\
\hline $\begin{array}{l}{[\text { Sexo }=2,00]{ }^{*}} \\
{[\text { Edadcategorica }=6,00]}\end{array}$ & $0^{a}$ & - & - & - & - & - \\
\hline $\begin{array}{l}{[\text { Sexo }=1,00]^{*}} \\
{[\text { Niveldeestudios }=1,00]}\end{array}$ & 1,011 & 0,027 & 37,175 & 0,000 & 0,958 & 1,065 \\
\hline $\begin{array}{l}{[\text { Sexo }=1,00]^{*}} \\
{[\text { Niveldeestudios }=2,00]}\end{array}$ & $-0,718$ & 0,006 & 125,267 & 0,000 & $-0,729$ & $-0,707$ \\
\hline $\begin{array}{l}{[\text { Sexo }=1,00]^{*}} \\
{[\text { Niveldeestudios }=3,00]}\end{array}$ & $-0,119$ & 0,002 & $-48,499$ & 0,000 & $-0,124$ & $-0,114$ \\
\hline $\begin{array}{l}{[\text { Sexo }=1,00]^{*}} \\
{[\text { Niveldeestudios }=4,00]}\end{array}$ & $-0,176$ & 0,002 & $-85,159$ & 0,000 & $-0,180$ & $-0,172$ \\
\hline $\begin{array}{l}{[\text { Sexo }=1,00]^{*}} \\
{[\text { Niveldeestudios }=5,00]}\end{array}$ & 0,150 & 0,002 & 63,111 & 0,000 & 0,145 & 0,155 \\
\hline $\begin{array}{l}{[\text { Sexo }=1,00]^{*}} \\
{[\text { Niveldeestudios }=6,00]}\end{array}$ & 0,202 & 0,002 & 87,142 & 0,000 & 0,197 & 0,206 \\
\hline $\begin{array}{l}{[\text { Sexo }=1,00]^{*}} \\
{[\text { Niveldeestudios }=7,00]}\end{array}$ & $-0,149$ & 0,002 & $-66,025$ & 0,000 & $-0,153$ & $-0,144$ \\
\hline $\begin{array}{l}{[\text { Sexo }=1,00]^{*}} \\
{[\text { Niveldeestudios }=8,00]}\end{array}$ & 0,142 & 0,002 & 61,989 & 0,000 & 0,138 & 0,147 \\
\hline $\begin{array}{l}{[\text { Sexo }=1,00]^{*}} \\
{[\text { Niveldeestudios }=9,00]}\end{array}$ & $0^{\mathrm{a}}$ & - & - & - & - & - \\
\hline $\begin{array}{l}{[\text { Sexo }=2,00]^{*}} \\
{[\text { Niveldeestudios }=1,00]}\end{array}$ & $0^{a}$ & - & - & - & - & - \\
\hline
\end{tabular}


Tabla 4 (continuación)

Estimaciones de los parámetros (modelo grupo $3^{\mathrm{d}}$ )

\begin{tabular}{|c|c|c|c|c|c|c|}
\hline \multirow{2}{*}{ Parámetro } & \multirow{2}{*}{ B } & \multirow{2}{*}{$\begin{array}{l}\text { Error } \\
\text { típ. }\end{array}$} & \multirow{2}{*}{$\mathbf{t}$} & \multirow{2}{*}{ Sig. } & \multicolumn{2}{|c|}{$\begin{array}{c}\text { Intervalo de confianza al } \\
95 \%\end{array}$} \\
\hline & & & & & $\begin{array}{l}\text { Límite } \\
\text { inferior }\end{array}$ & $\begin{array}{l}\text { Límite } \\
\text { superior }\end{array}$ \\
\hline $\begin{array}{l}{[\text { Sexo }=2,00]{ }^{*}} \\
{[\text { Niveldeestudios }=2,00]}\end{array}$ & $0^{a}$ & - & - & - & - & - \\
\hline $\begin{array}{l}{[\text { Sexo }=2,00]^{*}} \\
{[\text { Niveldeestudios }=3,00]}\end{array}$ & $0^{a}$ & - & - & - & - & - \\
\hline $\begin{array}{l}{[\text { Sexo }=2,00]{ }^{*}} \\
{[\text { Niveldeestudios }=4,00]}\end{array}$ & $0^{a}$ & - & - & - & - & - \\
\hline $\begin{array}{l}{[\text { Sexo }=2,00]} \\
{[\text { Niveldeestudios }=5,00]}\end{array}$ & $0^{a}$ & - & - & - & - & - \\
\hline $\begin{array}{l}{[\text { Sexo }=2,00]{ }^{*}} \\
{[\text { Niveldeestudios }=6,00]}\end{array}$ & $0^{a}$ & - & - & - & - & - \\
\hline $\begin{array}{l}{[\text { Sexo }=2,00]{ }^{*}} \\
{[\text { Niveldeestudios }=7,00]}\end{array}$ & $0^{a}$ & - & - & - & - & - \\
\hline $\begin{array}{l}{[\text { Sexo }=2,00]{ }^{*}} \\
{[\text { Niveldeestudios }=8,00]}\end{array}$ & $0^{\mathrm{a}}$ & - & - & - & - & - \\
\hline $\begin{array}{l}{[\text { Sexo }=2,00] \text { * }} \\
{[\text { Niveldeestudios }=9,00]}\end{array}$ & $0^{a}$ & - & - & - & - & - \\
\hline GSmotivacion & 0,235 & 0,000 & 1285,892 & 0,000 & 0,235 & 0,236 \\
\hline GSactividad & 0,281 & 0,000 & 1248,748 & 0,000 & 0,280 & 0,281 \\
\hline GSorg.trabajo & 0,171 & 0,000 & 988,956 & 0,000 & 0,170 & 0,171 \\
\hline GSsalario & 0,148 & 0,000 & 1025,557 & 0,000 & 0,148 & 0,148 \\
\hline
\end{tabular}

a. Al parámetro se le ha asignado el valor cero porque es redundante.

Nota: Variable dependiente: GStrabajoactual.

Fuente: Elaboración propia a partir de ECVT (Ministerio de Trabajo e Inmigración, 2010).

Ya que afirmamos que las variables propias del trabajo u organizacionales explican mejor la satisfacción laboral, en el modelo siguiente incluimos únicamente este tipo de variables pero en mayor número; para establecer una jerarquía entre ellas, utilizamos el método de regresión lineal múltiple stepwise, porque cuando existe un elevado número de posibles variables independientes conviene saber cuáles son las variables relevantes. Sólo se incorporan las variatablables que contribuyan de forma significativa al ajuste del modelo. La contribución de cada variable se establece contrastando, a partir del coeficiente de correlación parcial, la hipótesis de independencia entre esa variable y la variable dependiente. En la Tabla 5 aparecen las medias y las desviaciones típicas tanto para la variable dependiente como para las 14 variables independientes, todas ellas cuantitativas (toman valores de 0 a 10). El tamaño muestral, al eliminar la falta de respuesta, es ahora de 5841 personas. La variable grado satisfacción con el salario -GSsalario- es la segunda con menor 
puntuación media, indicativo del efecto de la situación de crisis económica actual.

Tabla 5

Estadísticos descriptivos

(variables propias del trabajo)

\begin{tabular}{|l|c|c|}
\hline \multicolumn{1}{|c|}{ Variables } & Media & Desviación típica \\
\hline GStrabajoactual & 7,3845 & 1,75104 \\
\hline GSposibilidadpromociones & 5,2229 & 3,18466 \\
\hline GSvaloracionsuperiores & 7,1555 & 2,20254 \\
\hline GSactividad & 7,7247 & 1,74923 \\
\hline GSdesarrollopersonal & 7,4884 & 1,97447 \\
\hline GSautonomia & 7,3143 & 2,18686 \\
\hline GSparticipaciondecisiones & 6,6269 & 2,62529 \\
\hline GSmotivacion & 7,0505 & 2,31005 \\
\hline GSjornada & 7,2256 & 2,25128 \\
\hline GSflexibilidadhorarios & 6,2988 & 3,08142 \\
\hline GSvacaciones,permisos & 7,4696 & 2,31126 \\
\hline GSestabilidad & 7,3655 & 2,53147 \\
\hline GSsalario & 5,8812 & 2,31639 \\
\hline GStiempodescanso & 6,7870 & 2,58370 \\
\hline GSorg.trabajo & 6,9721 & 2,13245 \\
\hline
\end{tabular}

Nota: variable dependiente: GStrabajoactual $\quad N=5841$

Fuente: Elaboración propia a partir de ECVT (Ministerio de Trabajo e Inmigración, 2010).

En este método stepwise se adopta el criterio de que una variable es incorporada al modelo si el nivel crítico asociado a su coeficiente de correlación parcial al contrastar la hipótesis de independencia es menor que 0,05 (probabilidad de entrada). Y queda fuera del modelo si ese nivel crítico es mayor que 0,10 (probabilidad de salida). De las 14 variables independientes el sistema elimina una, que es GSpartcipaciondecisiones.

La Tabla 6 recoge el valor de $\mathrm{R}, \mathrm{R}^{2}$ en cada paso. El error típico de la estimación va disminuyendo (pasa de 1,30559 a 1,05987). Una forma de valorar el efecto resultante de aplicar estos criterios de selección consiste en observar el cambio que se va produciendo en $\mathrm{R}^{2}$ a medida que se van incorporando variables al modelo. Un cambio grande en $\mathrm{R}^{2}$ indica que esa variable (en este caso GSmotivación) contribuye de forma importante a explicar lo que ocurre con la variable dependiente. En la tabla también aparece el estadístico F, que contrasta la hipótesis de que el cambio en $\mathrm{R}^{2}$ es cero en la población, y el nivel crítico 
asociado al estadístico F. Las 13 variables independientes explican un $63,4 \%$ de la varianza de la variable GStrabajoactual. El estadístico de Durbin-Watson vale 1,968 con lo que se asume independencia entre los residuos; éste es uno de los supuestos en que se basa el modelo de regresión lineal.

Tabla 6

Resumen del modelo en los distintos pasos ${ }^{\mathrm{n}}$

\begin{tabular}{|c|c|c|c|c|c|c|c|c|c|c|}
\hline \multirow[b]{2}{*}{ Modelo } & \multirow[b]{2}{*}{$\mathbf{R}$} & \multirow[b]{2}{*}{$\begin{array}{c}\mathbf{R} \\
\text { cuadrado }\end{array}$} & \multirow[b]{2}{*}{$\begin{array}{c}\mathbf{R} \\
\text { cuadrado } \\
\text { corregida }\end{array}$} & \multirow[b]{2}{*}{$\begin{array}{l}\text { Error típ. } \\
\text { de la } \\
\text { estimación }\end{array}$} & \multicolumn{5}{|c|}{ Estadísticos de cambio } & \multirow[b]{2}{*}{$\begin{array}{l}\text { Durbin- } \\
\text { Watson }\end{array}$} \\
\hline & & & & & $\begin{array}{c}\text { Cambio } \\
\text { en } R \\
\text { cuadrado }\end{array}$ & $\begin{array}{c}\text { Cambio } \\
\text { en F }\end{array}$ & gl1 & gl2 & $\begin{array}{l}\text { Sig. del } \\
\text { cambio } \\
\text { en F }\end{array}$ & \\
\hline 1 & $0,666^{\mathrm{a}}$ & 0,444 & 0,444 & 1,30559 & 0,444 & 4665,915 & 1 & 5839 & 0,000 & - \\
\hline 2 & $0,723^{\mathrm{b}}$ & 0,523 & 0,523 & 1,20913 & 0,079 & 969,844 & 1 & 5838 & 0,000 & - \\
\hline 3 & $0,753^{c}$ & 0,566 & 0,566 & 1,15343 & 0,043 & 578,477 & 1 & 5837 & 0,000 & - \\
\hline 4 & $0,771^{d}$ & 0,595 & 0,595 & 1,11489 & 0,029 & 411,432 & 1 & 5836 & 0,000 & - \\
\hline 5 & $0,781^{\mathrm{e}}$ & 0,610 & 0,609 & 1,09451 & 0,015 & 220,386 & 1 & 5835 & 0,000 & - \\
\hline 6 & $0,786^{f}$ & 0,619 & 0,618 & 1,08209 & 0,009 & 135,759 & 1 & 5834 & 0,000 & - \\
\hline 7 & $0,791^{9}$ & 0,626 & 0,626 & 1,07127 & 0,008 & 119,426 & 1 & 5833 & 0,000 & - \\
\hline 8 & $0,793^{h}$ & 0,629 & 0,629 & 1,06711 & 0,003 & 46,528 & 1 & 5832 & 0,000 & - \\
\hline 9 & $0,794^{i}$ & 0,631 & 0,630 & 1,06443 & 0,002 & 30,391 & 1 & 5831 & 0,000 & - \\
\hline 10 & $0,795^{j}$ & 0,632 & 0,632 & 1,06246 & 0,001 & 22,700 & 1 & 5830 & 0,000 & - \\
\hline 11 & $0,796^{k}$ & 0,634 & 0,633 & 1,06104 & 0,001 & 16,631 & 1 & 5829 & 0,000 & - \\
\hline 12 & $0,796^{\prime}$ & 0,634 & 0,633 & 1,06043 & 0,000 & 7,645 & 1 & 5828 & 0,006 & - \\
\hline 13 &, $0797^{\mathrm{m}}$ & 0,634 & 0,634 & 1,05987 & 0,000 & 7,207 & 1 & 5827 & 0,007 & 1,968 \\
\hline
\end{tabular}

a. Variables predictoras: (Constante), GSmotivacion

b. Variables predictoras: (Constante), a la anterior se añade GSactividad

c. Variables predictoras: (Constante), a las anteriores se añade GSorg.trabajo

d. Variables predictoras: (Constante), a las anteriores se añade GSsalario

e. Variables predictoras: (Constante), a las anteriores se añade GSjornada

f. Variables predictoras: (Constante), a las anteriores se añade GSvaloracionsuperiores

g. Variables predictoras: (Constante), a las anteriores se añade GSestabilidad

h. Variables predictoras: (Constante), a las anteriores se añade GSdesarrollopersonal

i. Variables predictoras: (Constante), a las anteriores se añade GSvacaciones,permisos

j. Variables predictoras: (Constante), a las anteriores se añade GSflexibilidadhorarios

k. Variables predictoras: (Constante), a las anteriores se añade GSautonomia

I. Variables predictoras: (Constante), a las anteriores se añade GStiempodescanso

$m$. Variables predictoras: (Constante), a las anteriores se añade GSposibilidadpromociones

n. Variable dependiente: GStrabajoactual

Fuente: Elaboración propia a partir de ECVT (Ministerio de Trabajo e Inmigración, 2010).

En la Tabla 7a aparecen los coeficientes correspondientes al modelo en el último paso. Los coeficientes estandarizados Beta proporcionan una información muy útil sobre la importancia relativa de cada variable independiente en la 
ecuación de regresión. Una variable tiene tanto más peso en la ecuación de regresión cuanto mayor (en valor absoluto) es su coeficiente Beta (el más alto corresponde a GSmotivación, 0,199). Los signos de los coeficientes $B$ son todos positivos, hecho ya comentado anteriormente. Las pruebas t y sus niveles críticos sirven para contrastar la hipótesis nula de que un coeficiente de regresión vale cero en la población. En todos los casos $\mathrm{p}<0,05$, con lo cual, los coeficientes son significativamente distintos de cero y, por tanto, contribuyen de forma significativa a explicar lo que ocurre con la variable GStrabajoactual.

Tabla 7a

Coeficientes de regresión parcial ${ }^{a}$ (modelo en último paso: 13 variables independientes)

\begin{tabular}{|l|c|c|c|c|c|}
\hline \multicolumn{1}{|c|}{ Modelo } & \multicolumn{2}{c|}{$\begin{array}{c}\text { Coeficientes no } \\
\text { estandarizados }\end{array}$} & $\begin{array}{c}\text { Coeficientes } \\
\text { estandarizados }\end{array}$ & t & Sig. \\
\hline (Constante) & B & Error típ. & Beta & 9,778 & 0,000 \\
\hline GSmotivacion & 0,728 & 0,074 & - & 16,326 & 0,000 \\
\hline GSactividad & 0,151 & 0,009 & 0,199 & 14,896 & 0,000 \\
\hline GSorg.trabajo & 0,197 & 0,013 & 0,196 & 13,931 & 0,000 \\
\hline GSsalario & 0,121 & 0,009 & 0,148 & 14,043 & 0,000 \\
\hline GSjornada & 0,096 & 0,007 & 0,127 & 7,626 & 0,000 \\
\hline GSvaloracionsuperiores & 0,060 & 0,008 & 0,077 & 9,673 & 0,000 \\
\hline GSestabilidad & 0,082 & 0,008 & 0,103 & 6,890 & 0,000 \\
\hline GSdesarrollopersonal & 0,047 & 0,007 & 0,067 & 5,830 & 0,000 \\
\hline GSvacaciones,permisos & 0,071 & 0,012 & 0,080 & 4,042 & 0,000 \\
\hline GSflexibilidadhorarios & 0,032 & 0,008 & 0,042 & 2,980 & 0,003 \\
\hline GSautonomia & 0,017 & 0,006 & 0,029 & 3,929 & 0,000 \\
\hline GStiempodescanso & 0,032 & 0,008 & 0,040 & 2,812 & 0,005 \\
\hline GSposibilidadpromociones & 0,019 & 0,007 & 0,028 & 2,685 & 0,007 \\
\hline
\end{tabular}

a. Variable dependiente: GStrabajoactual.

Fuente: Elaboración propia a partir de ECVT (Ministerio de Trabajo e Inmigración, 2010).

El nivel de tolerancia de una variable se obtiene restando a 1 el coeficiente de determinación $\mathrm{R}^{2}$ obtenido al regresar esa variable sobre el resto de variables independientes. Valores de tolerancia pequeños (próximos a cero) indican que esa variable puede ser explicada por una combinación lineal del resto de variables (multicolinealidad). Los factores de inflación de la varianza (FIV) son los inversos de los niveles de tolerancia. Cuanto mayor es el FIV de una variable, mayor será la varianza del coeficiente de regresión; por ello, un problema de multicolinealidad (tolerancias pequeñas, FIV grandes) es la inestabilidad de las 
estimaciones de los coeficientes de regresión. En la Tabla 7b las tolerancias no son pequeñas $y$, por tanto, no se deriva un problema de multicolinealidad.

Junto con los coeficientes de correlación parcial y semiparcial están las correlaciones de orden cero, que son las correlaciones de cada variable con GStrabajoactual (sin tener en cuenta la presencia de terceras variables). Se vuelve a comprobar que GSmotivacion es la variable que tiene la mayor correlación con GStrabajoactual. También se añade la matriz de correlaciones (véase Tabla 8); se puede comprobar que las correlaciones de orden cero (Tabla $7 \mathrm{~b}$ ) coinciden lógicamente con las correlaciones de la primera columna de esta tabla.

\section{Tabla $7 b$}

Correlaciones y niveles de tolerancia ${ }^{a}$ (modelo en último paso: 13 variables independientes)

\begin{tabular}{|c|c|c|c|c|c|}
\hline \multirow{2}{*}{ Modelo } & \multicolumn{3}{|c|}{ Correlaciones } & \multicolumn{2}{|c|}{$\begin{array}{l}\text { Estadísticos de } \\
\text { multicolinealidad }\end{array}$} \\
\hline & $\begin{array}{l}\text { Orden } \\
\text { cero }\end{array}$ & Parcial & Semip. & Tolerancia & FIV \\
\hline (Constante) & - & - & - & - & - \\
\hline GSmotivacion & 0,666 & 0,209 & 0,129 & 0,423 & 2,364 \\
\hline GSactividad & 0,636 & 0,192 & 0,118 & 0,361 & 2,769 \\
\hline GSorg.trabajo & 0,571 & 0,180 & 0,110 & 0,558 & 1,791 \\
\hline GSsalario & 0,453 & 0,181 & 0,111 & 0,771 & 1,297 \\
\hline GSjornada & 0,434 & 0,099 & 0,060 & 0,620 & 1,614 \\
\hline GSvaloracionsuperiores & 0,552 & 0,126 & 0,077 & 0,555 & 1,802 \\
\hline GSestabilidad & 0,413 & 0,090 & 0,055 & 0,655 & 1,526 \\
\hline GSdesarrollopersonal & 0,617 & 0,076 & 0,046 & 0,334 & 2,997 \\
\hline GSvacaciones, permisos & 0,416 & 0,053 & 0,032 & 0,581 & 1,722 \\
\hline GSflexibilidadhorarios & 0,369 & 0,039 & 0,024 & 0,644 & 1,553 \\
\hline GSautonomia & 0,497 & 0,051 & 0,031 & 0,612 & 1,633 \\
\hline GStiempodescanso & 0,394 & 0,037 & 0,022 & 0,618 & 1,618 \\
\hline GSposibilidadpromociones & 0,367 & 0,035 & 0,021 & 0,758 & 1,320 \\
\hline
\end{tabular}

a. Variable dependiente: GStrabajoactual

Fuente: Elaboración propia a partir de ECVT (Ministerio de Trabajo e Inmigración, 2010).

En la Tabla 9 se observa la mejora que se va produciendo en el modelo desde el paso 1 al 13 (último) al ir incorporando las variables. Se va incrementando la suma de cuadrados de la regresión en los distintos pasos, mientras que la suma de cuadrados residual va disminuyendo. También está el estadístico $\mathrm{F}$ obtenido al contrastar la hipótesis de que el valor de $\mathrm{R}^{2}$ en cada paso es cero. Si 
$\mathrm{R}^{2}$ es significativamente distinto de cero en el primer paso, también lo será en los pasos sucesivos.

Tabla 8

Matriz de correlaciones

\begin{tabular}{|c|c|c|c|c|c|c|c|c|c|c|c|c|c|c|c|}
\hline Variables & 1 & 2 & 3 & 4 & 5 & 6 & 7 & 8 & 9 & 10 & 11 & 12 & 13 & 14 & 15 \\
\hline 1. GStrabajoactual ${ }^{a}$ & 1 & & & & & & & & & & & & & & \\
\hline 2.GSposibilidadpromociones & 0,37 & 1 & & & & & & & & & & & & & \\
\hline 3. GSvaloracionsuperiores & 0,55 & 0,4 & 1 & & & & & & & & & & & & \\
\hline 4. GSactividad & 0,64 & 0,27 & 0,44 & 1 & & & & & & & & & & & \\
\hline 5. GSdesarrollopersonal & 0,62 & 0,32 & 0,44 & 0,78 & 1 & & & & & & & & & & \\
\hline 6. GSautonomia & 0,5 & 0,29 & 0,4 & 0,5 & 0,54 & 1 & & & & & & & & & \\
\hline 7.GSparticipaciondecisiones & 0,45 & 0,33 & 0,4 & 0,42 & 0,45 & 0,61 & 1 & & & & & & & & \\
\hline 8. GSmotivacion & 0,67 & 0,36 & 0,54 & 0,62 & 0,66 & 0,53 & 0,56 & 1 & & & & & & & \\
\hline 9. GSjornada & 0,43 & 0,16 & 0,28 & 0,34 & 0,33 & 0,27 & 0,22 & 0,34 & 1 & & & & & & \\
\hline 10. GSflexibilidadhorarios & 0,37 & 0,24 & 0,27 & 0,27 & 0,27 & 0,32 & 0,3 & 0,32 & 0,48 & 1 & & & & & \\
\hline 11. GStiempodescanso & 0,4 & 0,18 & 0,29 & 0,31 & 0,29 & 0,29 & 0,26 & 0,33 & 0,47 & 0,49 & 1 & & & & \\
\hline 12. GSvacaciones,permisos & 0,42 & 0,18 & 0,28 & 0,33 & 0,32 & 0,31 & 0,26 & 0,34 & 0,44 & 0,37 & 0,47 & 1 & & & \\
\hline 13. GSestabilidad & 0,41 & 0,21 & 0,29 & 0,31 & 0,31 & 0,32 & 0,3 & 0,33 & 0,36 & 0,3 & 0,33 & 0,54 & 1 & & \\
\hline 14. GSsalario & 0,45 & 0,28 & 0,32 & 0,3 & 0,31 & 0,28 & 0,28 & 0,37 & 0,31 & 0,23 & 0,28 & 0,3 & 0,31 & 1 & \\
\hline 15. GSorg.trabajo & 0,57 & 0,39 & 0,58 & 0,45 & 0,44 & 0,36 & 0,38 & 0,54 & 0,3 & 0,26 & 0,29 & 0,27 & 0,28 & 0,32 & 1 \\
\hline
\end{tabular}

a. Variable dependiente: GStrabajoactual,

Todas las correlaciones son significativas ( $p<0,001$ unilateral)

Fuente: Elaboración propia a partir de ECVT (Ministerio de Trabajo e Inmigración, 2010).

Tabla 9

Resumen del ANOVA ${ }^{\mathrm{n}}$

(pasos en la regresión stepwise)

\begin{tabular}{|c|c|c|c|c|c|c|}
\hline & Modelo & Suma de cuadrados & gl & Media cuadrática & $\mathbf{F}$ & Sig. \\
\hline \multirow{3}{*}{1} & Regresión & 7953,377 & 1 & 7953,377 & 4665,915 & $0,000^{\mathrm{a}}$ \\
\hline & Residual & 9952,983 & 5839 & 1,705 & - & - \\
\hline & Total & 17906,361 & 5840 & - & - & 一 \\
\hline \multirow{3}{*}{2} & Regresión & 9371,277 & 2 & 4685,639 & 3204,978 & $0,000^{b}$ \\
\hline & Residual & 8535,084 & 5838 & 1,462 & 一 & 一 \\
\hline & Total & 17906,361 & 5840 & - & - & 一 \\
\hline \multirow{3}{*}{3} & Regresión & 10140,877 & 3 & 3380,292 & 2540,829 & $0,000^{\mathrm{C}}$ \\
\hline & Residual & 7765,484 & 5837 & 1,330 & - & 一 \\
\hline & Total & 17906,361 & 5840 & - & - & - \\
\hline \multirow{3}{*}{4} & Regresión & 10652,282 & 4 & 2663,071 & 2142,475 & $0,000^{d}$ \\
\hline & Residual & 7254,079 & 5836 & 1,243 & - & 一 \\
\hline & Total & 17906,361 & 5840 & - & - & - \\
\hline \multirow{3}{*}{5} & Regresión & 10916,295 & 5 & 2183,259 & 1822,489 & $0,000^{\mathrm{e}}$ \\
\hline & Residual & 6990,066 & 5835 & 1,198 & - & - \\
\hline & Total & 17906,361 & 5840 & - & - & - \\
\hline
\end{tabular}




\section{Tabla 9 (continuación)}

Resumen del ANOVA ${ }^{\mathrm{n}}$

(pasos en la regresión stepwise)

\begin{tabular}{|c|c|c|c|c|c|c|}
\hline & Modelo & Suma de cuadrados & gl & Media cuadrática & $\mathbf{F}$ & Sig. \\
\hline \multirow{3}{*}{6} & Regresión & 11075,257 & 6 & 1845,876 & 1576,442 & $0,000^{f}$ \\
\hline & Residual & 6831,104 & 5834 & 1,171 & 一 & 一 \\
\hline & Total & 17906,361 & 5840 & - & - & - \\
\hline \multirow{3}{*}{7} & Regresión & 11212,312 & 7 & 1601,759 & 1395,726 & $0,000^{g}$ \\
\hline & Residual & 6694,049 & 5833 & 1,148 & - & 一 \\
\hline & Total & 17906,361 & 5840 & - & - & 一 \\
\hline \multirow{3}{*}{8} & Regresión & 11265,295 & 8 & 1408,162 & 1236,609 & $0,000^{h}$ \\
\hline & Residual & 6641,066 & 5832 & 1,139 & - & 一 \\
\hline & Total & 17906,361 & 5840 & - & 一 & 一 \\
\hline \multirow{3}{*}{9} & Regresión & 11299,729 & 9 & 1255,525 & 1108,124 & $0,000^{i}$ \\
\hline & Residual & 6606,632 & 5831 & 1,133 & - & 一 \\
\hline & Total & 17906,361 & 5840 & - & - & - \\
\hline \multirow{3}{*}{10} & Regresión & 11325,353 & 10 & 1132,535 & 1003,293 & $0,000^{j}$ \\
\hline & Residual & 6581,008 & 5830 & 1,129 & - & - \\
\hline & Total & 17906,361 & 5840 & - & - & - \\
\hline \multirow{3}{*}{11} & Regresión & 11344,076 & 11 & 1031,280 & 916,042 & $0,000^{k}$ \\
\hline & Residual & 6562,285 & 5829 & 1,126 & - & - \\
\hline & Total & 17906,361 & 5840 & - & - & - \\
\hline \multirow{3}{*}{12} & Regresión & 11352,673 & 12 & 946,056 & 841,300 & $0,000^{\prime}$ \\
\hline & Residual & 6553,688 & 5828 & 1,125 & - & - \\
\hline & Total & 17906,361 & 5840 & - & - & - \\
\hline \multirow{3}{*}{13} & Regresión & 11360,769 & 13 & 873,905 & 777,966 & $0,000^{m}$ \\
\hline & Residual & 6545,592 & 5827 & 1,123 & - & - \\
\hline & Total & 17906,361 & 5840 & - & - & - \\
\hline
\end{tabular}

a. Variables predictoras: (Constante), GSmotivacion

b. Variables predictoras: (Constante), a la anterior se añade GSactividad

c. Variables predictoras: (Constante), a las anteriores se añade GSorg.trabajo

d. Variables predictoras: (Constante), a las anteriores se añade GSsalario

e. Variables predictoras: (Constante), a las anteriores se añade GSjornada

f. Variables predictoras: (Constante), a las anteriores se añade GSvaloracionsuperiores

g. Variables predictoras: (Constante), a las anteriores se añade GSestabilidad

h. Variables predictoras: (Constante), a las anteriores se añade GSdesarrollopersonal

i. Variables predictoras: (Constante), a las anteriores se añade GSvacaciones,permisos

j. Variables predictoras: (Constante), a las anteriores se añade GSflexibilidadhorarios

k. Variables predictoras: (Constante), a las anteriores se añade GSautonomia

I. Variables predictoras: (Constante), a las anteriores se añade GStiempodescanso

$m$. Variables predictoras: (Constante), a las anteriores se añade GSposibilidadpromociones

n. Variable dependiente: GStrabajoactual

Fuente: Elaboración propia a partir de ECVT (Ministerio de Trabajo e Inmigración, 2010). 


\section{CONCLUSIONES}

La satisfacción laboral es un tema debatido tanto por sociólogos, como por economistas, psicólogos, y en cualquier área de conocimiento que afecte al ámbito laboral, sectores y ocupaciones. A la vista del marco teórico sobre el tema, se puede afirmar que los factores que pueden influir en la satisfacción de los trabajadores son muchos: las características individuales del trabajador, el contexto laboral y empresarial, factores ambientales, hasta el contexto macroeconómico y político general.

En este trabajo, utilizando los microdatos de la ECVT del año 2010, aplicamos distintas metodologías: modelos lineales generales (ANOVA) y modelo de regresión lineal múltiple stepwise, con la finalidad de explicar la variable GStrabajoactual. El objetivo inicial es averiguar si son las características personales de los trabajadores las que influyen en mayor grado en la satisfacción laboral o si, por el contrario, son las características inherentes al trabajo las que explican mejor la satisfacción en el trabajo. Con la aplicación de modelos lineales (ANOVA) se llegó a la conclusión de que aunque las variables son significativas igualmente, son las variables referidas al trabajo las que proporcionan mejores resultados en la explicación de la satisfacción laboral. Hay estudios empíricos que llegan a esa misma conclusión, como el de Mpeka (2012) -aunque las variables no sean exactamente las mismas. También hemos constatado que las mujeres presentan una puntuación en GStrabajoactual mayor que los hombres y que la relación entre la edad y GStrabajoactual asemeja forma de U (resultados coherentes con el marco teórico); con respecto al nivel de estudios no encontramos un patrón definido.

En el análisis hecho con la regresión lineal múltiple stepwise (14 variables independientes, todas referidas al trabajo) solo una fue eliminada por el sistema y de las otras 13, la que aporta mayor explicación a la variable GStrabajoactual es la motivación. En la regresión stepwise, los coeficientes de regresión son todos positivos, lo que se interpreta que al aumentar en un punto el grado de satisfacción en cualquiera de las variables inherentes al trabajo (sea actividad, salario, etc.) aumenta en B puntos -siendo B el coeficiente de regresión- la variable GStrabajoactual, manteniendo constantes las otras variables.

De los resultados de distintos estudios se deduce que a mayor satisfacción mayor rendimiento laboral, y por tanto, tendencia a mejorar la productividad del trabajo y el grado de competitividad de la empresa. Todo ello debería hacer reflexionar tanto a empresas como a instituciones públicas y privadas en el sentido de contemplar distintas medidas encaminadas a fomentar el avance en este tipo de satisfacción. Estas actuaciones podrían concretarse en cursos de formación dirigidos a empresarios y empleadores con la finalidad de mejorar la organización en el trabajo, en los turnos, permisos, etc. También serían de provecho cursos impartidos por personal especializado (psicólogos, sociólogos) dirigidos 
a empresarios y personal que ocupan puestos de dirección, en aras a mejorar la motivación y el desarrollo personal de sus subordinados, en definitiva, para que el clima laboral mejore. Mandar y motivar a la vez no es tarea fácil, y, si se consigue, además de reflejar una buena calidad profesional y humana, reportará beneficios al trabajador ya que estará más satisfecho, a la empresa por su mayor rendimiento, e incluso al directivo por la satisfacción de un trabajo bien hecho.

En la situación económica actual en la que se recortan salarios (en este estudio vimos que GSsalario es la segunda variable con menor puntuación media), aumenta la jornada de trabajo, proliferan los empleos precarios, contratos temporales, etc., no podemos esperar que el grado de satisfacción en el trabajo mejore, más bien al contrario. El análisis empírico expuesto tiene plena vigencia, ya que corresponde al momento de crisis económica española actual. Sin embargo, un estudio comparativo en distintos años serviría para conocer mejor la influencia que genera un período de contracción económica en la satisfacción laboral.

\section{REFERENCIAS BIBLIOGRÁFICAS}

ALLEN, J. Y VAN DER VELDEN, R. (2001): "Educational mismatches versus skill mismatches: Effects on wages, job satisfaction, and on-the-job search" en Oxford Economic Papers, Vol. 53, № 3: pp. 434-452.

ALONSO, M. A. (2004): "Centralidad del trabajo y metas en el trabajo: Dos variables claves en orientación laboral” en Revista de Educación, № 335: pp. 319-344.

BARMBY, T., BRYSON, A. Y EBERTH, B. (2012): "Human capital, matching and job satisfaction" en Economics Letters, Vol. 117, № 3: pp. 548-551.

BÒRIA-REVERTER, S., CRESPI-VALLBONA, M., Y MASCARILLA-MIRÓ, O. (2012): "Variables determinantes de la satisfacción laboral en España" en Cuadernos de Economía, Vol. 35, № 97: pp. 9-16.

BOWLING, N. A. (2010): "Effects of job satisfaction and conscientiousness on extra-role behaviors" en Journal of Business and Psychology, Vol. 25, № 1: pp. 119-130.

CABALLERO, K. (2002): "El concepto de satisfacción en el trabajo y su proyección en la enseñanza" en Profesorado, revista de currículum y formación del profesorado, Vol. $6, N^{\circ}(1-2)$.

CLARK, A., OSWALD, A. Y WARR, P. (2011): "Is job satisfaction U-shaped in age?" en Journal of Occupational and Organizational Psychology, Vol. 69, № 1: pp. 57-81.

COHRS, C., ABELE, A. E. Y DETTE, D. E. (2006): "Integrating situational and dispositional determinants of job satisfaction: Findings from three samples of professionals" en The Journal of Psychology, Vol. 140, № 4: pp. 363-995.

CUADRA-PERALTA, A. A. Y VELOSO-BESIO, C. B. (2010): "Grado de supervisión como variable moderadora entre liderazgo y satisfacción, motivación y clima organizacional" en Ingeniare. Revista chilena de ingeniería, Vol. 18, № 1: pp. 15-25. 
DEL LÍBANO, M., GARCÍA, M., LLORENS, S. Y SALANOVA, M. (2005): “ ¿Existen relaciones significativas entre adicción al trabajo y satisfacción?" en Jornades de Foment de la Investigació, Universitat Jaume I (España).

DONOHUE, S. M. Y HEYWOOD, J. S. (2004): "Job satisfaction and gender: an expanded specification from the NLSY" en International Journal of Manpower, Vol. 25, $N^{\circ} 2$ : pp. 211-238.

ECVT (2010): Encuesta de calidad de vida en el trabajo, Madrid: Ministerio de Trabajo e Inmigración.

ELLICKSON, M. C. Y LOGSDON, K. (2001): "Determinants of job satisfaction of municipal government employees" en State and Local Government Review, Vol. 33, № 3: pp. 173-184.

FRANEK, M. Y VECERA, J. (2008): "Personal characteristics and job satisfaction" en E+M Ekonomie a Management, № 4: pp. 63-76.

GAMERO, C. (2005): Análisis microeconómico de la satisfacción laboral, Consejo Económico y Social.

GAMERO, C. (2007): "Satisfacción laboral y tipo de contrato en España" en Investigaciones Económicas, Vol. 31, № 3: pp. 415-444.

GAMERO, C. (2010): "Satisfacción laboral de los asalariados inmigrantes" en Revista de Economía Aplicada, Vol. 18, № 54: pp. 33-56.

GAZIOGLU, S. Y TANSEL, A. (2006): "Job satisfaction in Britain: individual and job related factors" en Applied Economics, Vol. 38, № 10: pp. 1163-1171.

GÓMEZ, M. A. (2010): "Calidad de vida laboral en empleados temporales del Valle de Aburrá - Colombia" en Revista Ciencias Estratégicas, Vol. 18, № 24: pp. 225-236.

HERZBERG, F., MAUSNER, B. Y SNYDERMAN, B. B. (1959): The motivation to work. $2^{\mathrm{a}}$ ed. New York: Wiley.

IVIE, INSTITUTO VALENCIANO DE INVESTIGACIONES ECONÓMICAS (2007): "Actitudes de los jóvenes en el trabajo" en Cuadernos de Capital Humano, № 80.

JUDGE, T. A., THORESEN, C. J., BONO, J. E. Y PATTON, G. K. (2001): "The job satisfaction-job performance relationship: A qualitative and quantitative review" en Psychological Bulletin, Vol. 127, № 3: pp. 376-407.

KHANIN, D., TUREL, O. Y MAHTO, R. V. (2012): "How to increase job satisfaction and reduce turnover intentions in the family firm: the family-business embeddedness perspective" en Family Business Review, Vol. 25, № 4: pp. 391-408.

KOUSTELIOS, A. D. (2001): "Personal characteristics and job satisfaction of Greek teachers" en International Journal of Educational Management, Vol. 15, № 7: pp. 354-358.

LAWLER, E. E. (1973): Motivation in work organizations, Monterrey: Brooks/Cole.

LAWLER, E. E. Y PORTER, L. W. (1967): "The effect of performance on job satisfaction" en Industrial Relations, Vol. 7, № 1: pp. 20-28.

LÓPEZ-ARAÚJO, B., OSCA SEGOVIA, A. Y PEIRÓ SILLA, J. M. (2007): "El papel modulador de la implicación con el trabajo en la relación entre el estrés y la satisfacción laboral" en Psicothema, Vol. 19, № 1: pp. 81-87.

LUND, D. B. (2003): "Organizational culture and job satisfaction" en Journal of Business and Industrial Marketing, Vol. 18, № 3: pp. 219-236. 
MPEKA, R. L. (2012): "A study to examine the determinants of job satisfaction for professional accountants in Tanzania" en Afro-Asian Journal of Finance and Accounting, Vol. 3, № 1: pp. 15-33.

OKPARA, J. O. (2004): "Personal characteristics as predictors of job satisfaction: an exploratory study of IT managers in a developing economy" en Information Technology and People, Vol. 17, № 3: pp. 327-338.

OKPARA, J. O. (2006): "The impact of personal characteristics on the job satisfaction of public sector managers in a developing economy: implications for personnel development" en African Journal of Business and Economics Research, Vol. 1, № 1: pp. 10-29.

PÉREZ RUBIO, J. A. (1997): "Motivación y satisfacción laboral: retrospectiva sobre sus formas de análisis" en Revista Española de Investigaciones Sociológicas, № 80: pp. 133-167.

PETRESCU, A. I. Y SIMMONS, R. (2008): "Human resource management practices and workers' job satisfaction" en International Journal of Manpower, Vol. 29, № 7: pp. 651-667.

ROBLES-GARCÍA, M., DIERSSEN-SOTOS, T., MARTÍNEZ-OCHOA, E., HERRERACARRAL, P., DÍAZ-MENDI, A. R. Y LLORCA-DÍAZ, J. (2005): "Variables relacionadas con la satisfacción laboral: Un estudio transversal a partir del modelo EFQM" en Gaceta Sanitaria, Vol. 19, № 2: pp. 127-134.

RODE, J. C. (2004): “Job satisfaction and life satisfaction revisited: A longitudinal test of an integrated model" en Human Relations, Vol. 57, № 9: pp. 1205-1230.

SÁNCHEZ, S. M., FUENTES, F. J. Y ARTACHO, C. (2007): "La perspectiva de género en el análisis de la satisfacción laboral: una aplicación empírica mediante modelos logit y probit" en Revista Cuadernos de Gestión, Vol. 7, № 2: pp. 55-67.

SÁNCHEZ SELLERO, M. C. (2012): "Distintas formas de participación en el mercado laboral en Galicia (España). Influencia del género" en Atlantic Review of Economics, $N^{\circ}$ 1: pp. 1-34.

SÁNCHEZ SELLERO, M. C. Y SÁNCHEZ SELLERO, P. (2013): "Efectos de factores personales y organizativos sobre la satisfacción laboral en España" en Anales de Economía Aplicada, № 27: pp. 293-314.

SINGH, P. Y LONCAR, N. (2010): "Pay satisfaction, job satisfaction and turnover intent" en Industrial Relations, Vol. 65, No 3: pp. 470-490.

TREMBLAY, M., CHÊNEVERT, D. Y HÉBERT, A. (2012): "Le rôle des conditions de travail dans la satisfaction et la loyauté des infirmières d'agence au Québec" en Industrial Relations, Vol. 67, N 3: pp. 477-504.

WESTOVER, J. H. Y TAYLOR, J. (2010): "International differences in job satisfaction: The effects of public service motivation, rewards and work relations" en International Journal of Productivity and Performance Management, Vol. 59, № 8: pp. 811-828.

WESTOVER, J. H. (2012a): "The job satisfaction-gender paradox revisited: A crossnational look at gender differences in job satisfaction, 1989-2005" en Journal of Global Responsibility, Vol. 3, № 2: pp. 263-277.

WESTOVER, J. H. (2012b): "Comparative welfare state impacts on work quality and job satisfaction: A cross-national analysis" en International Journal of Social Economics, Vol. 39, № 7: pp. 503-525. 
ZEYTINOGLU, I., DENTON, M., DAVIES, S., BAUMANN, A., BLYTHE, J. Y BOOS, L. (2007): "Associations between work intensification, stress and job satisfaction: the case of nurses in Ontario" en Industrial Relations, Vol. 62, № 2: pp. 201-225. 